\title{
BMJ Open Effect of epidural analgesia on cancer outcomes after gastric cancer resection: a single-centre cohort study in Taiwan
}

\author{
Fu-Kai Hsu, ${ }^{1,2}$ Wen-Kuei Chang, ${ }^{1,2}$ Kuan-Ju Lin, ${ }^{1,2}$ Tan-Ju Chu, ${ }^{1,2}$ \\ Wen-Liang Fang, ${ }^{2,3}$ Kuang-Yi Chang (i) ${ }^{1,2}$
}

To cite: Hsu F-K, Chang W$\mathrm{K}$, Lin K-J, et al. Effect of epidural analgesia on cancer outcomes after gastric cancer resection: a single-centre cohort study in Taiwan. BMJ Open 2022;12:e053050. doi:10.1136/ bmjopen-2021-053050

- Prepublication history and additional supplemental material for this paper are available online. To view these files, please visit the journal online (http://dx.doi.org/10.1136/ bmjopen-2021-053050).

F-KH and W-KC contributed equally.

Received 03 May 2021

Accepted 12 February 2022

Check for updates

(C) Author(s) (or their employer(s)) 2022. Re-use permitted under CC BY-NC. No commercial re-use. See rights and permissions. Published by BMJ.

1Department of Anesthesiology, Taipei Veterans General Hospital, Taipei, Taiwan

${ }^{2}$ School of Medicine, National Yang Ming Chiao Tung University, Taipei, Taiwan

${ }^{3}$ Department of Surgery, Taipei Veterans General Hospital, Taipei, Taiwan

Correspondence to

Dr Kuang-Yi Chang;

kychang@vghtpe.gov.tw

\section{ABSTRACT}

Objective To investigate the influence of epidural anaesthesia and analgesia (EA) on cancer recurrence and overall survival after surgery for gastric cancer.

Study design and setting A retrospective study which involved patients with stage I-III gastric cancer undergoing curative resection in a medical centre from January 2012 to December 2017 and followed up until December 2019 through electronic medical chart review. Patient demographics, anaesthetic and surgical characteristics and pathologic features were also gathered.

Primary and secondary outcome measures The effects of EA on postoperative cancer recurrence and overall survival were evaluated using proportional hazards regression models with inverse probability of treatment weighting (IPTW). Multivariable Cox regression analyses were conducted for sensitivity analysis as well.

Results Among the 413 patients with median follow-up of 38.5 months (IQR: 22.1-59.7), 66 (16.0\%) received EA after gastric cancer surgery. EA was not associated with greater cancer recurrence (IPTW-adjusted HR: $0.55,95 \% \mathrm{Cl}: 0.27$ to $1.13, \mathrm{p}=0.102$ ) or cancer specific (IPTW- adjusted HR: 0.53, 95\% Cl: 0.27 to $1.04, \mathrm{p}=0.07$ ) and all-cause mortality (IPTW-adjusted HR: $0.65,95 \% \mathrm{Cl}$ : 0.37 to $1.16, p=0.143$ ) after gastric cancer resections. For sensitivity analysis, multivariable Cox regression analysis also generated non-significant EA effects on cancer recurrence and survival after surgery.

Conclusions There was no significant association between EA and cancer recurrence or overall survival in patients with stage I-III gastric cancer receiving surgical resection of primary tumour. Prospective study should be considered to elucidate the relationship between EA and cancer outcomes after gastric cancer surgery.

\section{INTRODUCTION}

Gastric cancer is the third most frequent cause of cancer mortality in the world, leading to around 800000 deaths each year. Surgical resection was the only possible curative treatment. ${ }^{2}$ Although tumour characteristics are the main factors related to gastric cancer recurrence, inflammatory responses may also play some roles in cancer outcomes after surgery. ${ }^{3-5}$ The secretion and synthesis of various inflammatory cytokine induced by surgical incision can lead to inflammatory

\section{Strengths and limitations of this study}

- This is the first study to investigate the effect of epidural anaesthesia and analgesia on oncological outcomes of patients with gastric cancer who underwent gastrectomy in Taiwan.

- Inverse probability of treatment weighting methodology which could avoid sample loss and compromise of statistical power was applied to reduce the imbalances in patient characteristics between groups and obtain more reliable estimation of epidural effects.

- Due to the nature of retrospective design, potential selection bias and unobserved confounding factors cannot be excluded.

responses. ${ }^{5}$ Besides, surgical intervention itself may cause tumour cell seeding into blood and lymphatic system. ${ }^{6}$ In spite of the dispersal of the tumour cell, the perioperative immunity can affect the development of metastasis. $^{7}$ The anaesthetic management, blood transfusion, hypothermia and opioid use may suppress the cell-mediated immunity and influence the microenvironment and growth of tumour cell. ${ }^{6}$

Previous study showed that the combination of epidural and general anaesthesia had increased viable T-lymphocyte and decreased inflammatory cytokines level compared with general anaesthesia only during the tumour resection of early-stage gastric cancer. $^{5}$ However, reviewing the recent literature, the role of epidural anaesthesia and analgesia (EA) in the prognosis of gastric cancer is still controversial. ${ }^{8-11}$ Accordingly, we designed this retrospective study to investigate the isolated impact of EA on recurrence and survival of patients undergoing curative resection of gastric cancer. We used inverse probability of treatment weighting (IPTW) based on propensity scores to weight study subjects by the probability of treatment and created a synthetic sample which eliminate imbalances in baseline characteristics between groups. ${ }^{12}$ 


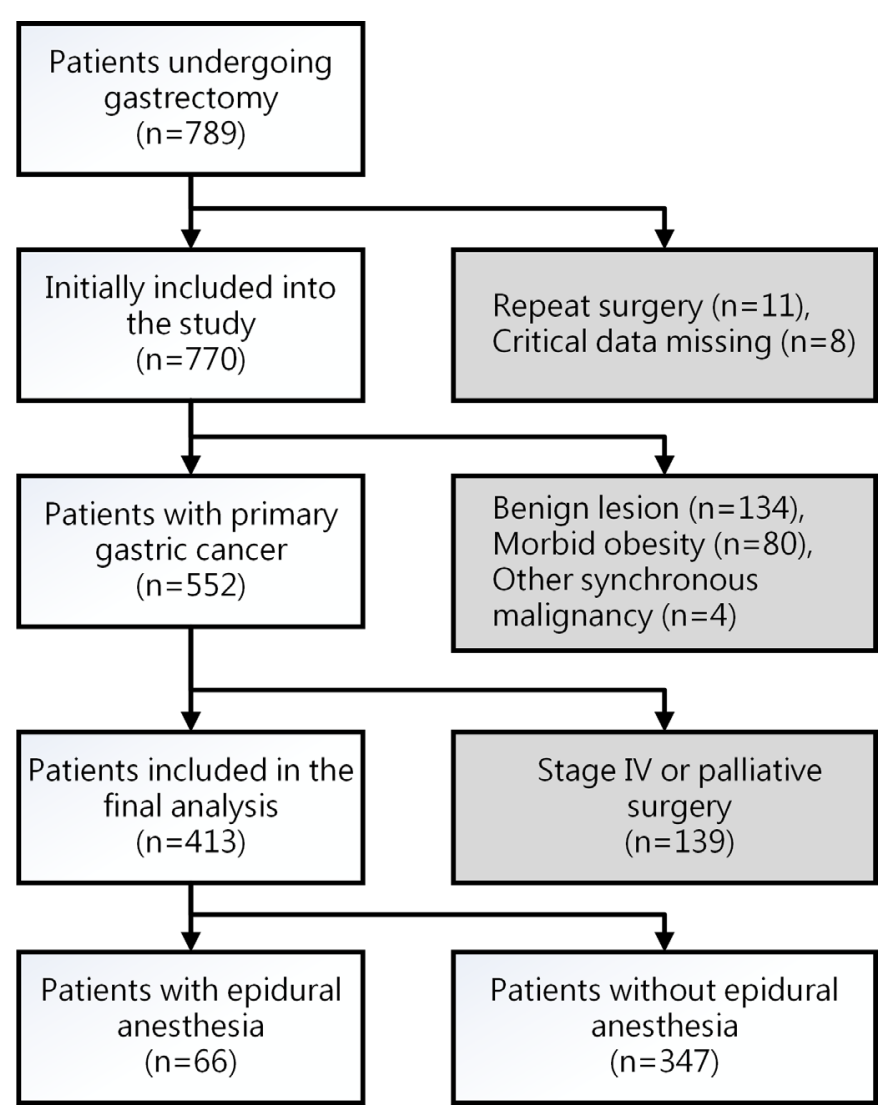

Figure 1 Flow diagram for patient selection.

In light of the positive associations in previous study, we hypothesise that EA may be associated with lower cancer recurrence rate and improved overall survival (OS) in patients receiving gastric cancer surgery. The risk factors of recurrence and mortality after gastric cancer surgery were also explored and their potentially confounding effects on cancer outcomes were further eliminated using sound analytical approaches.

\section{METHOD \\ Patient selection}

This study was approved and exempted from the need for patient consents by the Taipei Veterans General Hospital Institutional Review Board (IRB-TPEVGH no. 2018-06-009CC). All methods were conducted in accordance with local guidelines and regulations at the Taipei Veterans General Hospital. We reviewed the institutional electronic medical database of all patients who underwent gastric surgery at our medical centre from January 2012 to December 2017. Patients with previous gastric surgery, missing critical data about demographics, pathology result or postoperative analgesic evaluation were excluded (figure 1). According to the pathological result, surgeries for benign lesions and patients with synchronous malignancy were also excluded. Besides, stage IV disease or patients undergoing palliative surgery were also excluded from the analysis. The remaining patients were analysed in the two groups: one received general anaesthesia combination with perioperative EA and the other received general anaesthesia only.

\section{Patient and public involvement}

No patient involved.

\section{Analgesic management}

All patients who received major abdominal surgery in our medical centre were offered the choice of EA for perioperative and postoperative analgesia. The epidural catheters were implanted at the lower thoracic spine on the day before the surgery. To test the function of the epidurals, one bolus of lidocaine $2 \%$ was given after catheter placement. A loading dose of lidocaine 2\% 60-100 mg was administrated before surgery began and then the continuous infusion of bupivacaine $0.25 \%$ or $0.5 \%$ at a rate of 5-10 mL/hour was given during surgery. For postoperative pain control, EA with continuous dose of fentanyl 1 $\mu \mathrm{g} / \mathrm{mL}$ and bupivacaine $0.1 \%$ at a rate $4-6 \mathrm{~mL} /$ hour and bolus dose of $2-2.5 \mathrm{~mL}$ with a lockout interval of $15-30$ min were typically used until 48-72 hours after surgery. For patients who refused epidurals implantation because of surgeon or patient preference and those not suitable due to the contraindications for epidurals, an intravenous patient-controlled analgesia was administrated by an ambulatory infusion pump (Gemstar Yellow, Hospira, Illinois, USA) to deliver continuous infusion of morphine at a rate of $0.5-1.0 \mathrm{mg} /$ hour and boluses dose of $1 \mathrm{mg}$ with a lockout time of 6 min for postoperative analgesia.

\section{Data collection}

The electronic medical records of the included patients were reviewed by a specialist anaesthesiologist who was not involved in data analysis and following variables were collected: age, sex, body mass index, Charlson Comorbidity Index, ${ }^{13}$ preoperative haemoglobin and albumin level; pretreatment carcinoembryonic antigen and carbohydrate antigen 19-9 (CA19-9) level, ${ }^{14}$ anaesthesia time, surgical technique (open or laparoscopic) and blood loss, perioperative packed red blood cell transfusion, diameter of tumours, tumour nodes metastasis staging, ${ }^{15}$ histological differentiation, lymphovascular invasion and postoperative adjuvant chemotherapy.

The date of death was determined according to medical record or death certificate. Recurrence was decided by the radiologists and general surgeons based on the imaging studies (eg, CT, MRI, bone scan and so on). The primary outcome was recurrence-free survival (RFS) and the secondary outcome was cancer-specific survival (CSS) and OS. For those without cancer recurrence or death, the survival time was regarded as the corresponding censored observations. Competing risk events were regarded as censoring in the analysis of CSS.

\section{Statistical analysis}

Comparisons of baseline attributes between epidural and non-epidural groups were performed using $\chi^{2}$ tests for categorical covariates and either t tests or Wilcoxon rank sum tests for continuous covariates, as appropriate. 
Kaplan-Meier method was applied to compare the RFS and OS curves between groups. Univariate Cox regression analysis was used to assess the effect of covariates on RFS or OS. To cope with the potential imbalance of measured confounders between two groups, propensity scores based on a list of patient characteristics were generated to estimate the probability of receiving epidurals (online supplemental file 1). An IPTW method on propensity score was used to eliminate possible confounding effects from the imbalances in collected variables. The inverse of estimated probability was then used for further weighted regression analysis and stabilised weights were employed to diminish the impact of large weights on analytical results. ${ }^{12}$ Continuous variables are presented as mean with SD and categorical variables are expressed as count with percentage. To reduce skewness of non-normal continuous variables, such as anaesthesia time and blood loss during surgery, logarithmic transformation was applied. To assess balance in the distributions of observed covariates between the two groups, standardised differences were conducted. Weighted Cox regression analysis was applied to examine the association between EA and cancer recurrence or OS based on IPTW. For sensitivity analysis, significant predictors of RFS or OS in univariate analysis were considered as candidates for stepwise model selection procedures in multivariable models. The association between EA and outcomes was further examined adjusting for the determined predictors of the multivariable models. The significance level for all hypotheses was 0.05 for a two-tailed test. All the statistical analyses were performed using SAS software, V.9.4 (SAS Institute, Cary, North Carolina, USA). We employed Schoenfeld's formula for the proportional hazards regression model to estimate the minimum requirement of sample size. ${ }^{16}$ Based on the previous study by Hiller et al, ${ }^{17}$ at least 354 subjects were needed to achieve a power of 0.8 given a type I error rate of 0.05 and the proportion of patients receiving EA in our study.

\section{RESULT}

Among the 413 patients included in the analysis, 66 $(16.0 \%)$ received EA for gastric cancer resection. The median follow-up interval for all patients was 38.5 (IQR: 22.1-59.7) months. Note that before IPTW, more patients receiving EA before 2015 and the EA group had less chance to receive laparoscopic surgery and more blood loss during surgery (table 1). However, after IPTW, these variables become more balanced between groups (table 1).

\section{Epidural analgesia and recurrence risk}

Note that EA was not associated with recurrence risk $(\mathrm{HR}=0.8, \mathrm{p}=0.5$, figure $2 \mathrm{~A})$ in the univariate analysis. Multivariable analysis identified five independent prognostic factors of cancer recurrence, including body mass index (BMI) (HR=0.93), CA19-9 (on base-2 logarithmic scale, $\mathrm{HR}=1.15$ ), blood loss during surgery (on base-2 logarithmic scale, HR=1.30), cancer stage (II vs I, $\mathrm{HR}=2.41$; III vs I, HR=5.06) and pathologic lymphovascular invasion $(\mathrm{HR}=2.45)$ (table 2). The effect of EA on cancer recurrence after gastric cancer surgery remained non-significant after the adjustment for these independent predictors (HR $=0.58,95 \%$ CI: 0.30 to 1.11$)$. After IPTW, weighted Cox regression model shows nonsignificant difference in the risk of cancer recurrence between groups $(\mathrm{HR}=0.55,95 \%$ CI: 0.27 to 1.13 , $\mathrm{p}=0.102$, figure 2B)

\section{Epidural analgesia and mortality risk}

No significant difference in OS after surgery was noted between groups in the univariate analysis $(\mathrm{HR}=0.97$, $\mathrm{p}=0.91$, figure 2C). Multivariable model identified six independent prognostic factors of OS, including BMI $(\mathrm{HR}=0.89)$, Charlson Comorbidity Index $(\mathrm{HR}=1.24)$, CA19-9 (on base-2 logarithmic scale, HR=1.15), laparoscopic surgery $(\mathrm{HR}=0.53)$, anaesthesia time (on base-2 logarithmic scale, $\mathrm{HR}=2.16$ ) and cancer stage (II vs I, $\mathrm{HR}=1.39$; III vs I, HR=3.11) (table 3). The effect of EA on OS after gastric cancer surgery remained non-significant after the adjustment for these significant predictors (HR=0.75, 95\% CI: 0.45 to 1.25$)$, similar to the results of weighted Cox regression model $(\mathrm{HR}=0.65,95 \%$ CI: 0.37 to $1.16, \mathrm{p}=0.14$, figure 2D) after IPTW. Moreover, EA was not significantly associated with CSS either (IPTWadjusted HR: $0.53,95 \%$ CI: 0.27 to 1.04 , $\mathrm{p}=0.07$ ).

\section{DISCUSSION}

To our knowledge, this is the first study to investigate the effect of EA on oncological outcomes of patients with gastric cancer who underwent gastrectomy in Taiwan. Our study did not support the hypothetical effects of EA on RFS or OS after gastric cancer surgery. Besides, recent randomised clinical studies did not support such associations either ${ }^{18-20}$ and more prospective studies are necessary to evaluate the effects of EA on oncological outcomes after gastric cancer surgery. From the methodological perspective, our study applied sound analytical approaches to generate reliable estimations which provide valuable information about the relationships between EA and oncological outcomes after surgery. In this study, we collected major oncological prognostic factors to reduce potential confounding effects on the estimation of epidural influence on outcomes of interest. ${ }^{314}$ Besides, since comorbidity is an important predictor of cancer survival, the commonly used Charlson Comorbidity Index was also considered in the analysis. ${ }^{21}$ Moreover, we used the IPTW methodology to reduce the imbalances in patient characteristics between groups and obtain more reliable estimation of epidural effects. With limited sample size, the IPTW approach could avoid sample loss and compromise of statistical power which commonly happened in the propensity score matching and provide more precise estimations. ${ }^{12} 22$ Finally, multivariable regression models were employed to ensure the 
Table 1 Patient demographics

\begin{tabular}{|c|c|c|c|c|c|c|}
\hline & \multicolumn{3}{|l|}{ Original data } & \multicolumn{3}{|l|}{ After IPTW } \\
\hline & $\begin{array}{l}\text { EA group } \\
(n=66)\end{array}$ & $\begin{array}{l}\text { Non-EA group } \\
(n=347)\end{array}$ & $\begin{array}{l}\text { Standardised } \\
\text { difference }\end{array}$ & $\begin{array}{l}\text { EA group } \\
(n=60)\end{array}$ & $\begin{array}{l}\text { Non-EA group } \\
(n=354)\end{array}$ & $\begin{array}{l}\text { Standardised } \\
\text { difference }\end{array}$ \\
\hline Age, year $>70$ & $50(75.8 \%)$ & 225 (64.8\%) & 24.1 & 35 (57.7\%) & 235 (66.5\%) & 18.2 \\
\hline Sex, male & 32 (48.5\%) & $168(48.4 \%)$ & 0.1 & $28(46.5 \%)$ & $169(47.8 \%)$ & 2.6 \\
\hline $\mathrm{BMI}, \mathrm{kg} / \mathrm{m}^{2}$ & $23.7 \pm 3.8$ & $23.7 \pm 3.5$ & 1.5 & $23.8 \pm 3.4$ & $23.7 \pm 3.5$ & 3.4 \\
\hline $\mathrm{CCl}$ & $5 \pm 2$ & $5 \pm 2$ & 2.7 & $5 \pm 2$ & $5 \pm 2$ & 10.7 \\
\hline Haemoglobin, g/dL & $12.2 \pm 2.2$ & $11.9 \pm 2.1$ & 15.5 & $11.8 \pm 2.8$ & $11.9 \pm 2.1$ & 4.7 \\
\hline Albumin, $\mathrm{g} / \mathrm{dL}$ & $3.9 \pm 0.3$ & $3.9 \pm 0.4$ & 18.7 & $3.9 \pm 0.4$ & $3.9 \pm 0.4$ & 8.0 \\
\hline $\begin{array}{l}\text { Pretreatment CA19-9, } \\
\mathrm{U} / \mathrm{mL}\end{array}$ & $2(1.8-3.2)$ & $2(1.5-3.8)$ & 1.4 & $2(1.7-3.2)$ & $2(1.5-3.8)$ & 10.4 \\
\hline Pretreatment CEA, $\mu \mathrm{g} / \mathrm{L}$ & $9(4.8-15.0)$ & $7(3.3-15.9)$ & 13.4 & $7(3.5-13)$ & $8(3.3-15.9)$ & 18.9 \\
\hline Blood transfusion & $14(21.2 \%)$ & $91(26.2 \%)$ & 11.8 & $16(26.2 \%)$ & $90(25.5 \%)$ & 1.7 \\
\hline Year & & & 79.8 & & & 0.4 \\
\hline$<2015$ & $56(84.8 \%)$ & $174(50.1 \%)$ & & $33(55.5 \%)$ & $197(55.7 \%)$ & \\
\hline$\geq 2015$ & $10(15.2 \%)$ & $173(49.9 \%)$ & & $27(44.5 \%)$ & $157(44.3 \%)$ & \\
\hline Laparoscopic surgery & $15(22.7 \%)$ & $146(42.1 \%)$ & 42.3 & $23(38.1 \%)$ & $138(39.0 \%)$ & 1.9 \\
\hline Blood loss, mL & $175(50-300)$ & $100(30-200)$ & 42.8 & $100(30-200)$ & $100(50-250)$ & 26.6 \\
\hline Anaesthesia time, $\min$ & $330(300-405)$ & $330(270-375)$ & 22.3 & 300 (270-390) & $330(270-390)$ & 6.3 \\
\hline Postoperative $\mathrm{C} / \mathrm{T}$ & $34(51.5 \%)$ & $140(40.3 \%)$ & 22.6 & 27 (45.8\%) & $150(42.3 \%)$ & 7.1 \\
\hline TNM stage & & & 23.0 & & & 7.6 \\
\hline I & $18(27.3 \%)$ & $148(42.7 \%)$ & & $23(38.2 \%)$ & $142(40.2 \%)$ & \\
\hline II & $21(31.8 \%)$ & $77(22.2 \%)$ & & $13(20.9 \%)$ & $84(23.7 \%)$ & \\
\hline III & 27 (40.9\%) & $122(35.2 \%)$ & & $24(40.9 \%)$ & $128(36.1 \%)$ & \\
\hline Tumour size, $\geq 5 \mathrm{~cm}$ & 27 (40.9\%) & $146(42.1 \%)$ & 2.4 & 27 (45.5\%) & $149(42.1 \%)$ & 7.0 \\
\hline $\begin{array}{l}\text { Histological } \\
\text { differentiation }\end{array}$ & & & 7.2 & & & 1.9 \\
\hline Well to moderate & $33(50.0 \%)$ & $161(46.4 \%)$ & & $29(47.7 \%)$ & $166(46.8 \%)$ & \\
\hline Poor & $33(50.0 \%)$ & $186(53.6 \%)$ & & $31(52.3 \%)$ & $188(53.2 \%)$ & \\
\hline Lymphovascular invasion & $33(50.0 \%)$ & $167(48.1 \%)$ & 3.7 & $35(58.0 \%)$ & $171(48.3 \%)$ & 19.5 \\
\hline
\end{tabular}

Values were mean \pm SD, counts (per cent) or median (IQR). Continuous variables were analysed with either $t$ tests or Wilcoxon rank sum tests, as appropriate; categorical variables were analysed with Pearson $\chi^{2}$ tests. Standardised difference is the difference in mean, proportion or rank divided by the pooled SE, expressed as percentage; imbalance is defined as absolute value greater than 20 (small effect size). BMI, body mass index; CA19-9, carbohydrate antigen 19-9; CCI, Charlson Comorbidity Index; CEA, carcinoembryonic antigen; C/T, chemotherapy; EA, epidural anaesthesia and analgesia; IPTW, inverse probability of treatment weighting; TNM, tumour, node, metastases.

consistency of estimated results and explore risk factors of cancer recurrence and OS after curative surgery for gastric cancer in the current study.

Although some scholars suggested regional anaesthesia and analgesia could reduce opioid use to improve prognosis after cancer surgery because opioids might suppress natural killer cell activity and increase metastatic progression, ${ }^{23}$ common problem like relatively small sample size after propensity score matching or potential selection bias due to no matching was often noted in these studies. ${ }^{101123}$ On the contrary, our study did not support any beneficial effect of EA on the prognosis of gastric cancer. Wang et al demonstrated no association between epidural use and long-term survival and Shin $e t$ aldisclosed postoperative EA use was not related to better recurrence or mortality rate. ${ }^{8} 9$ The recent retrospective studies revealed no advantage of using EA in patients who underwent gastric cancer resection either. ${ }^{8}{ }^{24}$ Moreover, several randomised clinical studies investigating the EA effects on oncological outcomes in miscellaneous cancer surgeries failed to identify any significant connection in EA and cancer prognosis as well. ${ }^{18-20}$ Our rigorously designed observational study provides new evidence to reject the hypothesis of association between EA and oncological outcomes after gastric cancer surgery.

Our study also identified other factors associated with prognosis of gastric cancer after the regression analyses, including BMI, cancer stage, and so on. Jang et al proposed a U-shaped relationship between BMI and prognosis of gastric cancer ${ }^{25}$ but our study revealed higher BMI was 

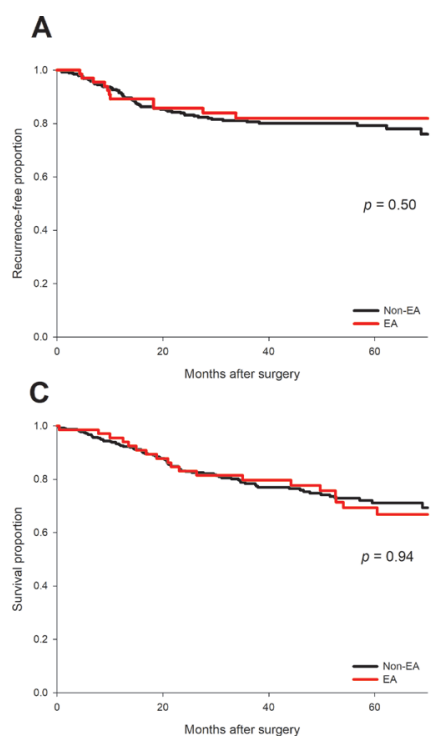

\section{B}

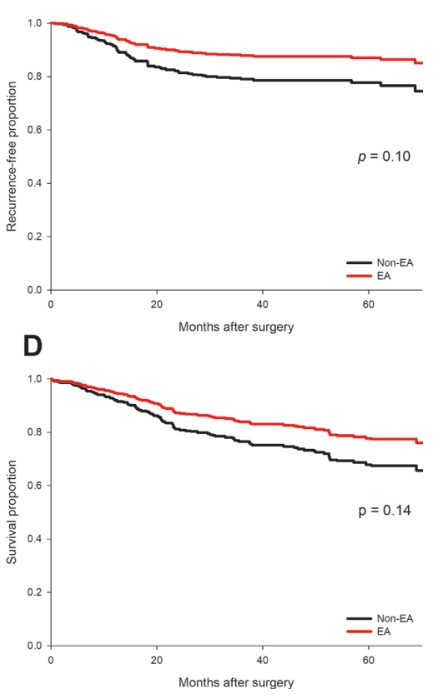

Figure 2 Kaplan-Meier curves for recurrence-free and overall survivals of the EA and non-EA groups in patients with gastric cancer surgery. No significant difference in cancer recurrence was noted before $(A)$ and after $(B)$ inverse probability treatment weighting. Besides, the differences in overall survival before (C) and after (D) inverse probability treatment weighting were not significant either. EA, epidural anaesthesia and analgesia.

associated with better oncological outcomes after gastric cancer surgery. Kambara et al demonstrated patients with excess body fatness tended to have higher surgical stress and postoperative infection rate but fewer lymph nodes dissection. By contrast, those with lower to normal range BMI were associated with worse immunity and nutritional status, and deeper tumour invasion. As a result, both the extremely high and low BMI might result in worse gastric cancer outcomes. ${ }^{26}$ Since most of the collected patients in our study were with low to normal range BMI, it is not strange to find higher BMI exhibited protective effects from inferior outcomes after gastric cancer surgery.

The previous studies have demonstrated both the laparoscopic group and open group had similar surgical

Table 2 Forward model selection for recurrence-free survival before weighting

\begin{tabular}{|c|c|c|c|}
\hline & HR & $95 \% \mathrm{Cl}$ & $\mathbf{P}$ \\
\hline Epidural analgesia & 0.58 & $0.30-1.11$ & 0.099 \\
\hline BMI & 0.93 & $0.87-0.99$ & 0.030 \\
\hline CA19-9* & 1.15 & $1.06-1.26$ & 0.001 \\
\hline Blood loss* & 1.30 & $1.10-1.52$ & 0.002 \\
\hline Cancer stage & & & $<0.001$ \\
\hline |I vs I & 2.41 & $0.96-6.07$ & 0.061 \\
\hline III vs I & 5.06 & $2.13-12.01$ & $<0.001$ \\
\hline Lymphovascular invasion & 2.45 & $1.32-4.53$ & 0.004 \\
\hline
\end{tabular}

${ }^{*}$ On base-2 logarithmic scale.

BMI, body mass index; CA19-9, carbohydrate antigen 19-9.
Table 3 Forward model selection for overall survival before weighting

\begin{tabular}{lllr}
\hline & HR & 95\% CI & P \\
\hline Epidural analgesia & 0.75 & $0.45-1.25$ & 0.266 \\
BMI & 0.89 & $0.84-0.94$ & $<0.001$ \\
$\begin{array}{l}\text { Charlson Comorbidity } \\
\text { Index }\end{array}$ & 1.24 & $1.12-1.37$ & $<0.001$ \\
\hline CA19-9* & 1.15 & $1.07-1.25$ & $<0.001$ \\
\hline Laparoscopic surgery & 0.53 & $0.33-0.85$ & 0.009 \\
\hline Anaesthesia time* & 2.16 & $1.29-3.64$ & 0.004 \\
\hline Cancer stage & & & $<0.001$ \\
\hline \multicolumn{1}{l}{ II vs I } & 1.39 & $0.75-2.60$ & 0.297 \\
\hline \multicolumn{1}{l|ll}{ vs I } & 3.11 & $1.85-5.24$ & $<0.001$ \\
\hline
\end{tabular}

${ }^{*}$ On base-2 logarithmic scale.

BMI, body mass index; CA19-9, carbohydrate antigen 19-9.

complication rate, anastomotic leakage, number of resected lymph nodes and oncological outcomes. ${ }^{27} 28$ However, in our study, it is noticed that patients receiving laparoscopic gastrectomy had better OS but similar RFS after controlling for the effects of other selected factors. It might result from the fact that patients with better general condition and less advanced disease had more chance to receive laparoscopic gastric cancer surgery ${ }^{29}$ and thus laparoscopic surgery was associated with better OS in our study.

Our study also demonstrated that blood loss during surgery is a risk factor for cancer recurrence. Intraoperative blood loss may cause immunosuppression by loss of plasma constituents. ${ }^{30}$ The decrease in natural killer cell activity was also noted after excessive blood loss. The intraoperative blood transfusion due to blood loss may increase interleukin- 6 and tumour growth factor. ${ }^{31}$ Besides, excessive bleeding may cause impaired wound healing and postoperative complications. ${ }^{32}$ Furthermore, the dispersion of microscopic cancer cells into pelvic cavity via blood loss may result in peritoneal recurrence. ${ }^{31}$ Nevertheless, the effects of intraoperative blood loss on cancer prognosis are inconclusive and deserve further investigations.

There are several limitations in our study. First, although potential selection bias and unobserved confounding effects may exist due to the retrospective design, we have included major prognostic factors to reduce the impacts from uncollected confounders. Second, cancer outcomes of patients lost to follow-up were difficult to collect and the corresponding censoring time was used in the analysis. Third, we did not collect the consumptions of opioids and non-opioid analgesics due to data unavailability. However, it is not necessary to include opioid demands in the analysis since the opioid-sparing effect is an advantage of EA and opioid consumption after surgery could be regarded as another endpoint of EA use, instead of a confounding factor in evaluating the effects of EA on 
cancer outcomes. Fourth, only $16 \%$ of patients received EA and most of them underwent surgery before 2015 and without laparoscopic surgery. However, the imbalances in the distributions of these two factors between the EA and non-EA groups improved considerably after the IPTW adjustment.

In conclusion, our study rejected the hypothetical association between EA and reduced cancer recurrence or all-cause mortality in patients with stage I-III gastric cancer undergoing curative surgery and provided useful information about the prognostic factors of postoperative cancer recurrence and survival. More prospective studies and randomised control trials should be considered in the future to elucidate the relationships between EA and oncological outcomes after gastric cancer surgery.

AcknowledgmentsThis study was supported by grants from the Taipei Veterans General Hospital, Taipei, Taiwan (V111C-074), the Ministry of Science and Technology, Taipei, Taiwan (MOST108-2511-H-075-001), Yen Tjing Ling Medical Foundation, Taipei, Taiwan (Cl-108-27) and the Anesthesiology Research and Development Foundation, Taipei, Taiwan (ARDF10804).

Contributors $\mathrm{F}-\mathrm{KH}, \mathrm{W}-\mathrm{KC}$ and $\mathrm{K}-\mathrm{YC}$ participated in conceptualisation. W-LF, $\mathrm{F}-\mathrm{KH}$ and $\mathrm{K}-\mathrm{JL}$ were responsible for data acquisition and interpretation. T-JC and $\mathrm{K}-\mathrm{YC}$ contributed to formal analysis, F-KH drafted the manuscript. W-KC and K-YC participated in manuscript revision and editing. $\mathrm{K}-\mathrm{YC}$, as guarantor, approved the final version of the manuscript for publication. All authors have read and agreed to the published version of the manuscript.

Funding This work was supported by grants from Yen Tjing Ling Medical Foundation (Cl-108-27), Anesthesiology Research and Development Foundation (ARDF10804), Ministry of Science and Technology (MOST108-2511-H-075-001), Taipei, Taiwan and Taipei Veterans General Hospital (V111C-074).

Competing interests None declared.

Patient consent for publication Not applicable.

Ethics approval This study does not involve human participants.

Provenance and peer review Not commissioned; externally peer reviewed.

Data availability statement Data are available upon reasonable request. The data that support the findings of this study are available from the corresponding author, upon reasonable request.

Supplemental material This content has been supplied by the author(s). It has not been vetted by BMJ Publishing Group Limited (BMJ) and may not have been peer-reviewed. Any opinions or recommendations discussed are solely those of the author(s) and are not endorsed by BMJ. BMJ disclaims all liability and responsibility arising from any reliance placed on the content. Where the content includes any translated material, BMJ does not warrant the accuracy and reliability of the translations (including but not limited to local regulations, clinical guidelines, terminology, drug names and drug dosages), and is not responsible for any error and/or omissions arising from translation and adaptation or otherwise.

Open access This is an open access article distributed in accordance with the Creative Commons Attribution Non Commercial (CC BY-NC 4.0) license, which permits others to distribute, remix, adapt, build upon this work non-commercially, and license their derivative works on different terms, provided the original work is properly cited, appropriate credit is given, any changes made indicated, and the use is non-commercial. See: http://creativecommons.org/licenses/by-nc/4.0/.

\section{ORCID iD}

Kuang-Yi Chang http://orcid.org/0000-0002-9062-5519

\section{REFERENCES}

1 Bray F, Ferlay J, Soerjomataram I, et al. Global cancer statistics 2018: GLOBOCAN estimates of incidence and mortality worldwide for 36 cancers in 185 countries. CA Cancer J Clin 2018;68:394-424.

2 Smyth EC, Nilsson M, Grabsch HI, et al. Gastric cancer. Lancet 2020;396:635-48.
3 Nakanishi Y, Ohara M, Domen H, et al. Differences in risk factors between patterns of recurrence in patients after curative resection for advanced gastric carcinoma. World J Surg Oncol 2013;11:98.

4 Nakagawa M, Kojima K, Inokuchi M, et al. Patterns, timing and risk factors of recurrence of gastric cancer after laparoscopic gastrectomy: reliable results following long-term follow-up. Eur $J$ Surg Oncol 2014;40:1376-82.

5 Liu W, Wu L, Zhang M, et al. Effects of general anesthesia with combined epidural anesthesia on inflammatory response in patients with early-stage gastric cancer undergoing tumor resection. Exp Ther Med 2019;17:35-40.

6 Tohme S, Simmons RL, Tsung A. Surgery for cancer: a trigger for metastases. Cancer Res 2017;77:1548-52.

7 Wu H-L, Tai Y-H, Chan M-Y, et al. Effects of epidural analgesia on cancer recurrence and long-term mortality in patients after nonsmall-cell lung cancer resection: a propensity score-matched study. BMJ Open 2019;9:e027618.

8 Wang J, Guo W, Wu Q, et al. Impact of combination epidural and general anesthesia on the long-term survival of gastric cancer patients: a retrospective study. Med Sci Monit 2016;22:2379-85.

9 Shin S, Kim H-I, Kim NY, et al. Effect of postoperative analgesia technique on the prognosis of gastric cancer: a retrospective analysis. Oncotarget 2017;8:104594-604.

10 Wang Y, Wang L, Chen $\mathrm{H}$, et al. The effects of intra- and postoperative anaesthesia and analgesia choice on outcome after gastric cancer resection: a retrospective study. Oncotarget 2017;8:62658-65.

11 Pei J-P, Zhang C-D, Liang Y, et al. Effects of epidural combined with general anesthesia versus general anesthesia alone in gastric cancer surgery: a propensity score matching analysis. Ann Trans/ Med 2020;8:473

12 Austin PC, Stuart EA. Moving towards best practice when using inverse probability of treatment weighting (IPTW) using the propensity score to estimate causal treatment effects in observational studies. Stat Med 2015;34:3661-79.

13 Charlson ME, Pompei P, Ales KL, et al. A new method of classifying prognostic comorbidity in longitudinal studies: development and validation. J Chronic Dis 1987;40:373-83.

$14 \mathrm{Kim}$ DH, Oh SJ, Oh CA, et al. The relationships between perioperative CEA, Ca 19-9, and Ca 72-4 and recurrence in gastric cancer patients after curative radical gastrectomy. J Surg Oncol 2011;104:585-91.

15 Amin MB, Greene FL, Edge SB, et al. The Eighth Edition AJCC Cancer Staging Manual: Continuing to build a bridge from a population-based to a more "personalized" approach to cancer staging. In: Ca: a cancer Journal for clinicians. , 2017: 67, 93-9

16 Schoenfeld DA. Sample-size formula for the proportional-hazards regression model. Biometrics 1983;39:499-503.

17 Hiller JG, Hacking MB, Link EK, et al. Perioperative epidural analgesia reduces cancer recurrence after gastro-oesophageal surgery. Acta Anaesthesiol Scand 2014;58:281-90.

18 Sessler DI, Pei L, Huang Y, et al. Recurrence of breast cancer after regional or general anaesthesia: a randomised controlled trial. Lancet 2019;394:1807-15

19 Falk W, Magnuson A, Eintrei C, et al. Comparison between epidural and intravenous analgesia effects on disease-free survival after colorectal cancer surgery: a randomised multicentre controlled trial. Br J Anaesth 2021;127:65-74.

20 Xu Z-Z, Li H-J, Li M-H, et al. Epidural Anesthesia-Analgesia and recurrence-free survival after lung cancer surgery: a randomized trial. Anesthesiology 2021;135:419-32.

21 Lin J-X, Huang Y-Q, Xie J-W, et al. Age-Adjusted Charlson comorbidity index (Accl) is a significant factor for predicting survival after radical gastrectomy in patients with gastric cancer. BMC Surg 2019;19:53

22 Tai YH, Wu HL, Mandell MS, et al. The association of allogeneic blood transfusion and the recurrence of hepatic cancer after surgical resection. Anaesthesia 2020;75:464-71.

23 Horowitz M, Neeman E, Sharon E, et al. Exploiting the critical perioperative period to improve long-term cancer outcomes. Nat Rev Clin Oncol 2015;12:213-26.

24 Cummings KC, Patel M, Htoo PT, et al. A comparison of the effects of epidural analgesia versus traditional pain management on outcomes after gastric cancer resection: a population-based study. Reg Anesth Pain Med 2014;39:200-7.

25 Jang J, Wang $\mathrm{T}$, Cai $\mathrm{H}$, et al. The $\mathrm{U}$-shaped association between body mass index and gastric cancer risk in the Helicobacter pylori biomarker cohort Consortium: a nested case-control study from eight East Asian cohort studies. Int J Cancer 2020;147:777-84.

26 Kambara Y, Yuasa N, Takeuchi E, et al. Overweight or obesity is an unfavorable long-term prognostic factor for patients who 
underwent gastrectomy for stage II/III gastric cancer. World $J$ Surg 2019;43:1766-76.

27 Cai J, Wei D, Gao CF, et al. A prospective randomized study comparing open versus laparoscopy-assisted D2 radical gastrectomy in advanced gastric cancer. Dig Surg 2011;28:331-7.

28 Park YK, Yoon HM, Kim Y-W, et al. Laparoscopy-Assisted versus open D2 distal gastrectomy for advanced gastric cancer: results from a randomized phase II multicenter clinical trial (COACT 1001). Ann Surg 2018;267:638-45.

29 Solsky I, In H. Surgical treatment for gastric cancer. Gastrointest Endosc Clin N Am 2021;31:581-605.
30 Bruns CJ, Schäfer $\mathrm{H}$, Wolfgarten B, et al. [Effect of intraoperative blood loss on the function of natural killer cells in tumors of the upper gastrointestinal tract]. Langenbecks Arch Chir Suppl Kongressbd 1996;113:146-9.

31 Nakanishi K, Kanda M, Kodera Y. Long-Lasting discussion: adverse effects of intraoperative blood loss and allogeneic transfusion on prognosis of patients with gastric cancer. World J Gastroenterol 2019;25:2743-51.

32 Mizuno A, Kanda M, Kobayashi D, et al. Adverse effects of intraoperative blood loss on long-term outcomes after curative gastrectomy of patients with stage II/III gastric cancer. Dig Surg 2016;33:121-8. 